\title{
Conversion therapy for inoperable advanced gastric cancer patients by docetaxel, cisplatin, and S-1 (DCS) chemotherapy: a multi-institutional retrospective study
}

\author{
Yasushi Sato $^{1} \cdot$ Hiroyuki Ohnuma $^{1} \cdot$ Takayuki Nobuoka $^{2} \cdot$ Masahiro Hirakawa $^{1} \cdot$ Tamotsu Sagawa $^{3}$. \\ Koshi Fujikawa $^{3}$ - Yasuo Takahashi ${ }^{3} \cdot$ Minami Shinya $^{4} \cdot$ Shinich Katsuki $^{5} \cdot$ Minoru Takahashi $^{6}$. $^{2}$ \\ Masahiro Maeda $^{7} \cdot$ Yutaka Okagawa $^{1} \cdot$ Uemura Naoki $^{1} \cdot$ Syouhei Kikuch $^{1} \cdot$ Koichi Okamoto $^{8}$. \\ Hiroshi Miyamoto $^{8} \cdot$ Mitsuo Shimada $^{9} \cdot$ Takemasa Ichiro $^{2} \cdot$ Junji Kato $^{1} \cdot$ Tetsuji Takayama $^{8}$
}

Received: 29 March 2016/ Accepted: 8 August 2016/Published online: 23 August 2016

(c) The International Gastric Cancer Association and The Japanese Gastric Cancer Association 2016

\begin{abstract}
Background Conversion therapy is an option for unresectable metastatic gastric cancer when distant metastases are controlled by chemotherapy; however, the feasibility and efficacy remain unclear. This study aimed to assess the feasibility and efficacy of conversion therapy in patients with initially unresectable gastric cancer treated with docetaxel, cisplatin, and S-1 (DCS) chemotherapy by evaluating clinical outcomes.
\end{abstract}

Yasushi Sato

yasushis2008@gmail.com

1 Department of Medical Oncology and Hematology, Sapporo Medical University School of Medicine, South 1 West 16, Chuo-ku, Sapporo 060-8543, Japan

2 Department of Surgery, Surgical Oncology and Science, Sapporo Medical University School of Medicine, Sapporo, Japan

3 Department of Gastroenterology, Hokkaido Cancer Center, Sapporo, Japan

4 Department of Gastroenterology, Oji General Hospital, Tomakomai, Japan

5 Department of Gastroenterology, Otaru Ekisaikai Hospital, Otaru, Japan

6 Department of Gastroenterology, Sapporo Kyoritsu Gorinbashi Hospital, Sapporo, Japan

7 Department of Gastroenterology, Steel Memorial Muroran Hospital, Muroran, Japan

8 Department of Gastroenterology and Oncology, Institute of Health Biosciences, The University of Tokushima Graduate School, Tokushima, Japan

9 Department of Digestive and Pediatric Surgery, Institute of Health Biosciences, The University of Tokushima Graduate School, Tokushima, Japan
Methods One hundred unresectable metastatic gastric cancer patients, enrolled in three DCS chemotherapy clinical trials, were retrospectively evaluated. The patients received oral $\mathrm{S}-1$ (40 $\mathrm{mg} / \mathrm{m}^{2}$ b.i.d.) on days $1-14$ and intravenous cisplatin $\left(60 \mathrm{mg} / \mathrm{m}^{2}\right)$ and docetaxel $\left(50-60 \mathrm{mg} / \mathrm{m}^{2}\right)$ on day 8 every 3 weeks. Conversion therapy was defined when the patients could undergo R0 resection post-DCS chemotherapy and were able to tolerate curative surgery.

Results Conversion therapy was achieved in 33/100 patients, with no perioperative mortality. Twenty-eight of the 33 patients $(84.8 \%)$ achieved $\mathrm{R} 0$ resection, and $78.8 \%$ were defined as histological chemotherapeutic responders. The median overall survival (OS) of patients who underwent conversion therapy was 47.8 months (95\% CI 28.0-88.5 months). Patients who underwent R0 resection had significantly longer OS than those who underwent R1 and $\mathrm{R} 2$ resections $(P=0.0002)$. Of the patients with primarily unresectable metastases, $10 \%$ lived $>5$ years. Among patients who underwent conversion therapy, multivariate analysis showed that the pathological response was a significant independent predictor for OS.

Conclusions DCS safely induced a high conversion rate, with very high R0 and pathological response rates, and was associated with a good prognosis; these findings warrant further prospective investigations.

Keywords Gastric cancer · Conversion therapy · DCS

\section{Introduction}

Gastric cancer is the second most common cause of cancer death worldwide [1] and has the highest incidence of any cancer in Japan. Surgical resection during the early stage has 
improved the treatment outcomes of localized gastric cancer, with long-term disease-free survival achieved in many cases $[2,3]$. However, many patients have recurrences or are diagnosed with distant metastasis unsuitable for curative surgery, and these patients have extremely poor prognoses $[4,5]$. For such patients, systemic chemotherapy is the only potential treatment; however, it is mainly administered to provide palliation and prolong survival. During the last decade, several new agents with promising activity against gastric cancer have been identified, including S-1, docetaxel, oxaliplatin, and irinotecan [6]. In western countries, the most commonly used treatments for unresectable metastatic gastric cancer are combination chemotherapy regimens comprising fluoropyrimidine [5-fluorouracil (5-FU) or an oral fluoropyrimidine] plus a platinum agent, although docetaxel or anthracyclines are sometimes combined [7, 8]. In Japan, S-1 plus cisplatin is currently recognized as a standard treatment for unresectable and metastatic gastric cancer with an overall survival (OS) of 13 months in the SPIRITS trial [9].

These developments have raised new clinical issues in the treatment of incurable gastric cancer. In some patients, incurable disease apparently disappears or is well controlled during primary chemotherapy. For such patients, surgery to excise any macroscopically remaining disease with curative intent may be possible. In fact, it has recently been reported that conversion from unresectable to resectable metastatic colorectal cancer through advances in systemic chemotherapy, termed "conversion therapy," can improve the prognosis [10] to a 5-year survival rate of $30-50 \%$ [11]. Conversion therapy is currently regarded as a standard modality in the multidisciplinary treatment of metastatic colorectal cancer patients.

However, the feasibility and efficacy of conversion therapy with curative surgery remain unclear in patients with unresectable metastatic gastric cancer. Conversion therapy is defined as surgical treatment aimed at R0 resection post-chemotherapy for tumors originally considered unresectable or marginally resectable for technical and/or oncological reasons [12]. This type of surgery for gastric cancer, also known as conversion surgery [13], secondary gastrectomy [14], or adjuvant surgery [15], appears potentially beneficial in terms of patient survival; however, it remains unclear whether such treatment can be conducted safely and with certainty and to what extent patient survival is prolonged. Moreover, the indications, most appropriate chemotherapy regimens, and timing of the operation remain to be clarified, as there is a paucity of information on the value of conversion therapy postchemotherapy in gastric cancer patients, mainly because of insufficient responses to various chemotherapy regimens.

We previously conducted phase I and II studies to evaluate the effect of adding docetaxel to base treatment with S-1 plus cisplatin (DCS) as a means to further improve the therapeutic response. Both a very high response rate $(87.1 \%)$ and a promising median survival time (MST; 687 days) in patients with unresectable advanced gastric cancer were noted [16, 17]. During these trials, we encountered patients whose responses were sufficient to undergo curative surgery after DCS chemotherapy. Furthermore, we reported that neoadjuvant treatment with DCS combination for locally advanced gastric cancer demonstrated a sufficient R0 resection rate and a good pathological response, with manageable toxicities [18]. Accordingly, DCS is considered a highly promising regimen for achieving conversion therapy for unresectable gastric cancer. The purpose of the present study was to examine the possibility of such conversion therapy by retrospectively examining the conversion rate and prognosis in patients with initially unresectable gastric cancer treated with the DCS regimen.

\section{Patients and methods}

\section{Selection criteria and treatments}

From December 2002 to April 2014, our group enrolled 100 gastric cancer patients in three consecutive trials [18, 36 , and 46 patients in the phase I study [16], phase II study of DCS [17], and phase II study of modified-dose DCS (UMIN000002361)]. The main selection criteria in all three trials were: (1) histologic confirmation of stomach adenocarcinoma; (2) unresectable distant metastatic disease [M1 stage, Japanese Classification of Gastric Carcinoma [19] (JGCA v.13)]; (3) measurable lesion(s); (4) age between 20-80 years; (5) Eastern Cooperative Oncology Group scale performance status $0-2$; (6) no prior chemotherapy; (7) adequate bone marrow, liver, and renal functions; and (8) provision of written informed consent. These studies were approved by the ethics committee of each institution and hospital. The treatment dosage and schedules were as follows: DCS (S-1 $40 \mathrm{mg} / \mathrm{m}^{2}$ b.i.d. on days $1-14$, cisplatin $60 \mathrm{mg} / \mathrm{m}^{2}$, docetaxel $60 \mathrm{mg} / \mathrm{m}^{2}$ on day 8 every 3 weeks), modified-dose DCS (as reported above, except for docetaxel at $50 \mathrm{mg} / \mathrm{m}^{2}$ ). These treatments were repeated until unacceptable toxicity, disease progression, patient refusal, or a response that enabled conversion therapy with curative operation was observed. Metastatic lesions (M1) were judged to be absent post-DCS chemotherapy according to the findings of conventional examinations such as computed tomography, ultrasonography, or magnetic resonance imaging. Further examinations using radionuclide bone scintigraphy and/or (18F)-fluoro-2-deoxy-D-glucose-positron emission tomography/computed tomography were 
performed, if clinically indicated, to exclude M1 disease. Staging laparoscopy was also performed as needed to exclude occult M1 disease in the peritoneum or other intraabdominal sites. Surgical resection was classified as curative when no evidence of disease was found after surgery. In downstaged patients, conversion therapy was considered when R0 resection was deemed possible by gastrectomy with more than $\mathrm{D} 2$ lymph node (LN) dissection. When the previous metastatic site included the para-aortic nodes, additional dissection of the para-aortic nodes was performed if possible. Non-downstaged patients whose lesions were judged to be curatively resectable by extended surgery (combined resection) were also considered for conversion therapy. In this process, a multidisciplinary team comprising medical oncologists and surgeons re-evaluated all potentially resectable cancers to define the best resection strategy. Conversion therapy was performed within 6 weeks of the last chemotherapy cycle. In this study, palliative surgery was not evaluated. Therefore, chemotherapy was continued if non-curative factors were recognized following assessment by laparoscopy or laparotomy. After conversion therapy, we restarted and continued chemotherapy at the attending physician's discretion (regimens with S-1 or docetaxel plus S-1 were most frequently used).

\section{Assessment and follow-up}

Toxicity was evaluated according to the Common Toxicity Criteria for Adverse Events (version 3.0). Responses were assessed after each cycle according to the response evaluation criteria in solid tumors guidelines (version 1.0) and for primary lesions according to the guidelines of the Japanese classification of gastric carcinoma [20]. The pathological response to chemotherapy was classified according to the following Japanese gastric cancer association criteria [21]: grade 0, no part of the tumor affected; grade 1a, less than one-third affected; grade $1 \mathrm{~b}$, between one-third and two-thirds affected; grade 2, more than twothirds affected; and grade 3, no residual tumor. Pathological response was defined as one-third or more of the tumor affected (grade 1b, 2, or 3). Postoperatively, all patients underwent computed tomography at least every 3 months during the first 3 years, followed by every 6 months until 5 years post-surgery.

\section{Statistics}

Patient characteristics and chemotherapy results were compared using the chi-square test for heterogeneity or with Fisher's exact test when appropriate. We calculated the cumulative OS rates by the Kaplan-Meier method and compared the survival curves with the log-rank test. OS was estimated from chemotherapy initiation to the date of death or the last follow-up. We subjected significant variables from the log-rank test $(P$ values $<0.05)$ to multivariate Cox proportional hazard regression analysis to assess the independence of the prognostic factors. In the multivariate analysis, we calculated the hazard ratio with $95 \%$ confidence intervals (CIs). All statistical analyses were performed using JMP software, version 8.0 (SAS Institute, Cary, NC), and $P$ values $<0.05$ were considered significant.

\section{Results}

\section{Clinicopathological characteristics and clinical results}

Patients' demographic characteristics are outlined in Table 1. The patients included 71 males $(71 \%)$ and 29 females (29\%) (median age, 63 years; range, 26-78 years). Most patients were in good general condition ( $74 \%$ had a performance status of 0 or 1). Histologically, 39 (39\%) patients had intestinal-type and 61 (61\%) had diffuse-type tumors. The reasons for unresectability included distant LN metastasis in $61(61 \%)$ patients (para-aortic LN, $n=59$; Virchow's LN, $n=5$; mediastinal LN $n=2$ ), peritoneal metastasis in 33 (33\%) (including 2 patients with positive peritoneal lavage cytology determined by staging laparoscopy), liver metastasis in $29(29 \%)$, bone metastasis in 6 $(6 \%)$, lung metastasis in $6(6 \%)$, ovary metastasis in 5 $(5 \%)$, and unresectable T4 lesions in 14 patients (14\%) (liver, $n=6$; colon, $n=5$; pancreas, $n=3$ ) with distant LN metastasis. Fifty-three patients had $>1$ factor indicating incurable tumors. A total of 642 chemotherapy cycles (median 6, range 1-12) were administered. The overall response rate was $81 \%$ (95\% CI 73.3-88.7\%) with 3 complete $(3 \%)$ and 78 partial responses $(78 \%)$. There were 19 cases of stable disease (19\%), but no case of progressive disease. The incidence rates of hematological grade $3 / 4$ adverse events were as follows: leukocytopenia $63 \%$, neutropenia $75 \%$, anemia $12 \%$, thrombocytopenia $10 \%$, and febrile neutropenia $12 \%$. Non-hematological grade 3 or higher adverse events included anorexia $(34.0 \%)$, nausea $(32.0 \%)$, and diarrhea $(14.0 \%)$. There were no chemotherapy-related deaths. All treatment-related toxicities were resolved with appropriate care, and no treatment-related deaths were observed.

\section{Clinical course to conversion therapy}

A flow diagram of the patients' treatment course is shown in Fig. 1. Thirty-three patients $(33 \%)$ achieved conversion therapy, including five patients who received second- 
Table 1 Patient characteristics and clinical results of 100 patients who underwent chemotherapy with DCS

\begin{tabular}{|c|c|}
\hline Characteristics & $N=100$ \\
\hline Age, years, median (range) & $63(26-78)$ \\
\hline Sex, male/female & $71 / 29$ \\
\hline Performance status, $(0 / 1 / 2)$ & $47 / 27 / 26$ \\
\hline Histology, ( intestinal/diffuse) & $39 / 61$ \\
\hline \multicolumn{2}{|l|}{ Tumor location } \\
\hline Upper third & 35 \\
\hline Middle third & 25 \\
\hline Lower third & 26 \\
\hline Whole body & 14 \\
\hline \multicolumn{2}{|l|}{ T stage (JGCA v.13) } \\
\hline $\mathrm{T} 2$ & 19 \\
\hline $\mathrm{T} 3$ & 67 \\
\hline $\mathrm{T} 4$ & 14 \\
\hline \multicolumn{2}{|l|}{$\mathrm{N}$ stage (JGCA v.13) } \\
\hline No & 3 \\
\hline N1 & 18 \\
\hline $\mathrm{N} 2$ & 18 \\
\hline N3 & 61 \\
\hline \multicolumn{2}{|l|}{ Distant metastases } \\
\hline Lymph node & 61 \\
\hline Peritoneum & 33 \\
\hline Liver & 29 \\
\hline Bone & 6 \\
\hline Lung & 6 \\
\hline Ovary & 5 \\
\hline \multicolumn{2}{|l|}{ Non-curative factors } \\
\hline $1 / \geq 2$ & $47 / 53$ \\
\hline \multicolumn{2}{|l|}{ DCS administration } \\
\hline Median number of courses (range) & $6(1-12)$ \\
\hline \multicolumn{2}{|l|}{ Response } \\
\hline Complete response & 3 \\
\hline Partial response & 78 \\
\hline Stable disease & 19 \\
\hline Progressive disease & 0 \\
\hline \multicolumn{2}{|l|}{ Adverse events (grade 3/4) } \\
\hline Leucopenia & 63 \\
\hline Neutropenia & 75 \\
\hline Anemia & 12 \\
\hline Thrombocytopenia & 10 \\
\hline Febrile neutropenia & 12 \\
\hline Anorexia & 34 \\
\hline Nausea & 32 \\
\hline Diarrhea & 14 \\
\hline
\end{tabular}

JGCA Japanese Gastric Cancer Association

line chemotherapy (CPT-11, $n=1$; CPT-11 plus cisplatin, $n=4)$. Thus, the conversion rate was $33 \%$ in this cohort. $1^{\text {st }}$ line DCS $(n=100)$

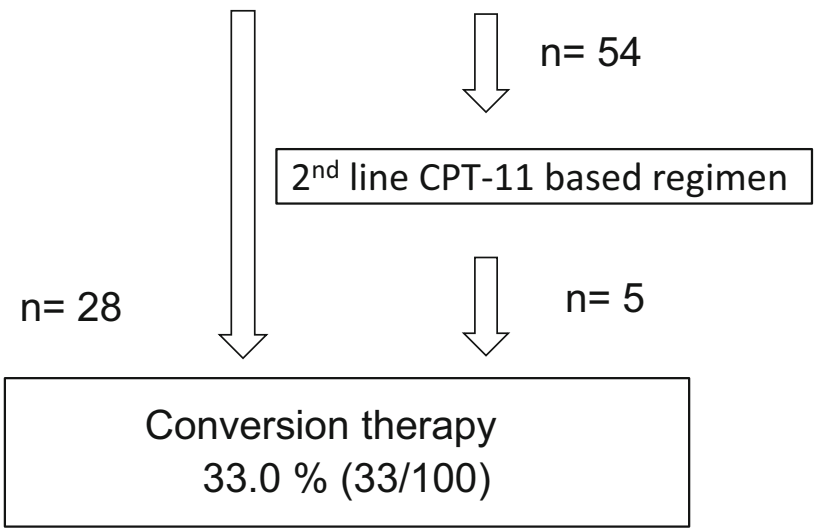

Fig. 1 Flow diagram of the patients' clinical course from docetaxel, cisplatin, and S-1 (DCS) chemotherapy to surgery

\section{Characteristics and chemotherapy results of the patients undergoing and not undergoing conversion therapy}

The demographic and clinical characteristics and chemotherapy results are summarized in Table 2 . In the patient characteristics at baseline, no significant differences were observed in terms of age, sex, histologic type, tumor location, $\mathrm{T}$ stage, nodal status, chemotherapy cycles, and distant metastasis between the two groups, whereas significant differences in performance status and a number of non-curative factors were noted. Among those who underwent conversion therapy, 32 patients (97\%) displayed a major response (2 complete and 30 partial responses), which was significantly better than in those who did not receive conversion therapy. In terms of treatment-related toxicities, no significant differences were observed between the groups.

\section{Surgery and pathologic results}

Among the 33 conversion therapy patients, R0 resection was performed in $28(84.8 \%)$ and $\mathrm{R} 1$ or $\mathrm{R} 2$ resection in 5 (positive peritoneal cytology in 2, unresectable ovarian metastasis, pancreas invasion, and peritoneal metastasis in 1 case each, respectively). Furthermore, total gastrectomy was performed in $29(87.9 \%)$ and distal gastrectomy in 4 (12.1\%) of these patients, who received more than D2 LN dissection (Table 3). Among the nine conversion cases with peritoneal metastasis, staging laparoscopy was performed in six cases, revealing the disappearance of the metastasis. Among the six patients with liver metastases, two underwent partial hepatectomies with a complete pathological response, and two were treated with radiofrequency ablation, after which the metastatic lesions 
Table 2 Results of conversion cases in comparison to non-conversion cases regarding the characteristics and chemotherapy results

\begin{tabular}{|c|c|c|c|}
\hline Characteristics & Conversion $(+)(n=33), n, \%$ & Conversion $(-)(n=67), n, \%$ & $P$ value \\
\hline Age, years, median (range) & $62(34-78)$ & $64(26-78)$ & 0.771 \\
\hline Sex, male/female & 22/11 (66.7/33.3) & 49/18 (73.1/26.9) & 0.639 \\
\hline Performance status, $(0 / 1 / 2)$ & $23 / 7 / 3(69.7 / 21.2 / 9.1)$ & $24 / 20 / 23(35.8 / 29.9 / 34.3)$ & 0.003 \\
\hline Histology, (intestinal/diffuse) & $13 / 20(39.4 / 60.6)$ & 26/41 (38.8/61.2) & 1.000 \\
\hline \multicolumn{4}{|l|}{ Tumor location } \\
\hline Upper third & $14(42.4)$ & $21(31.3)$ & \multirow[t]{4}{*}{0.304} \\
\hline Middle third & $8(24.2)$ & $17(25.4)$ & \\
\hline Lower third & $5(15.2)$ & $21(31.3)$ & \\
\hline Whole body & $6(18.2)$ & $8(11.9)$ & \\
\hline \multicolumn{4}{|l|}{ T stage (JGCA v.13) } \\
\hline $\mathrm{T} 2$ & $7(21.2)$ & $12(17.9)$ & \multirow[t]{3}{*}{0.271} \\
\hline $\mathrm{T} 3$ & $19(57.6)$ & 48 (71.6) & \\
\hline $\mathrm{T} 4$ & $7(21.2)$ & $7(10.4)$ & \\
\hline \multicolumn{4}{|l|}{$\mathrm{N}$ stage (JGCA v.13) } \\
\hline No & $1(3.0)$ & $2(3.0)$ & \multirow[t]{4}{*}{0.406} \\
\hline N1 & $5(15.1)$ & $13(19.4)$ & \\
\hline $\mathrm{N} 2$ & $9(27.3)$ & $9(13.4)$ & \\
\hline $\mathrm{N} 3$ & $18(54.5)$ & $43(64.2)$ & \\
\hline \multicolumn{4}{|l|}{ Distant metastases } \\
\hline $\mathrm{L} / \mathrm{N}$ & $16(48.5)$ & $45(67.2)$ & 0.084 \\
\hline Liver & $6(18.2)$ & $23(34.3)$ & 0.107 \\
\hline Peritoneum & $9(27.3)$ & $24(35.8)$ & 0.499 \\
\hline Bone & $2(6.1)$ & $4(6.0)$ & 1 \\
\hline Lung & $1(3.0)$ & $5(7.5)$ & 0.661 \\
\hline Ovary & $2(6.1)$ & $3(4.5)$ & 1 \\
\hline \multicolumn{4}{|l|}{ Non-curative factor } \\
\hline $1 / \geqq 2$ & $24 / 9(72.7 / 27.3)$ & $23 / 44(34.3 / 65.7)$ & $<0.001$ \\
\hline \multicolumn{4}{|l|}{ DCS administration } \\
\hline Median number of courses (range) & $4(2-12)$ & $5(1-11)$ & 0.364 \\
\hline \multicolumn{4}{|l|}{ Response } \\
\hline Complete response & $2(6.1)$ & $1(1.5)$ & \\
\hline Partial response & $30(90.9)$ & $48(71.6)$ & \\
\hline Stable disease & $1(3.0)$ & $18(26.9)$ & \\
\hline Progressive disease & $0(0)$ & $0(0)$ & \\
\hline Response rate $(\%)$ & 97.0 & 73.1 & 0.003 \\
\hline \multicolumn{4}{|l|}{ Adverse events (grade 3/4) } \\
\hline Neutropenia & $24(72.7)$ & $51(76.1)$ & 0.807 \\
\hline Leucopenia & $24(72.7)$ & $39(58.2)$ & 0.189 \\
\hline Anemia & $4(12.1)$ & $8(11.9)$ & 1.000 \\
\hline Thrombocytopenia & $3(9.1)$ & 7 (21.2) & 1.000 \\
\hline Febrile neutropenia & $2(6.1)$ & $10(14.9)$ & 0.327 \\
\hline Anorexia & $7(21.2)$ & $27(40.3)$ & 0.074 \\
\hline Nausea & $8(24.2)$ & $24(35.8)$ & 0.265 \\
\hline Diarrhea & $4(12.1)$ & $10(14.9)$ & 1.000 \\
\hline
\end{tabular}

JGCA Japanese Gastric Cancer Association 
Table 3 Surgical and pathological findings of 33 patients who underwent conversion therapy

\begin{tabular}{lll}
\hline & $n$ & $\%$ \\
\hline Operative procedure & & \\
Total gastrectomy & 29 & 87.9 \\
Distal gastrectomy & 4 & 12.1 \\
Combined resections & & \\
Liver & 4 & 12.1 \\
Pancreas & 2 & 6.1 \\
Colon & 1 & 3.0 \\
Resection margin & & \\
R0 & 28 & 84.8 \\
R1/R2 & 5 & 15.2 \\
Pathological response (primary site) & & \\
1a & 7 & 21.2 \\
1b & 10 & 30.3 \\
2 & 11 & 33.3 \\
3 & 5 & 15.2 \\
Complications & & \\
Wound infection & 2 & 6.1 \\
Abdominal abscess & 1 & 3.0 \\
Abdominal fluid collection & 2 & 6.1 \\
Pancreatic fistula & 1 & 3.0 \\
Leakage & 2 & 0.1 \\
30/60 day mortality & $0 / 0$ & \\
\hline
\end{tabular}

completely disappeared. Extensive resections were performed in T4 tumors estimated to have invaded the lateral segment of the liver, pancreas, and transverse colon in four, two, and one patient(s), respectively. The pathologic response rate of the primary tumors was $78.8 \%$, which included grade $1 \mathrm{a}, 1 \mathrm{~b}, 2$, and 3 in $7,10,11$, and 5 patients, respectively. Postoperative complications were observed in eight patients $(24.2 \%)$. Mortality and serious complications were not observed (Table 3 ).

\section{Postoperative chemotherapy}

In this study, 28/33 patients received adjuvant chemotherapy after surgery. S-1-based regimens were selected in 25 patients ( $\mathrm{S}-1$ alone in $18, \mathrm{~S}-1$ plus docetaxel in $4, \mathrm{~S}-1$ plus cisplatin in 2 , and DCS in 1 patient) and CPT-11 in 3 patients. The median number of postoperative S-1 chemotherapy courses was 8 (range 2-8), and the 1 -year completion rate was $66.7 \%(12 / 18)$.

\section{Survival}

Overall survival curves are shown in Fig. 2. The MST for all patients was 21.7 months at a median follow-up duration of 20.7 months (range 16-137.3 months) (Fig. 2a). In the 33 conversion therapy patients, the MST reached 47.8 months (95\% CI 28.0-88.5 months), with 1-, 3-, and 5-year OS rates of 97.0, 63.6, and $42.4 \%$, respectively, whereas the MST was 15.7 months $(95 \%$ CI 12.5-18.8 months) and the 1-, 3-, and 5-year OS rates were $65.9,18.7$, and $0 \%$, respectively, for the 67 patients who did not achieve conversion therapy (Fig. 2b). During follow-up, recurrence was observed in 17/33 patients treated with conversion therapy. Recurrent sites included the peritoneum $(n=8)$, LNs $(n=4)$, lungs $(n=1)$, liver $(n=3)$, and brain and lungs $(n=1)$. Ten patients survived $>5$ years after conversion therapy. Among the 33 patients treated with surgery, 28 patients $(84.8 \%)$ who underwent R0 resection exhibited a 5-year OS rate of $48.6 \%$ (MST, 47.9 months), while 5 patients who underwent R1 and R2 resections exhibited a rate of $0 \%$ (MST 21.7 months) (Fig. 2c). Accordingly, R0 resection led to significantly longer $\mathrm{OS}$ than $\mathrm{R} 1$ and $\mathrm{R} 2$ resections $(P=0.0002)$. Among the 61 patients with distant LN metastases, LN involvement was the only incurable factor in 27 patients; of these, 9 patients $(33.3 \%, 9 / 27)$ achieved conversion therapy. In these patients, the recurrence rate was $30 \%$, with the MST not yet reached. DCS treatment led to conversions therapy in 6 patients among 29 patients $(20.7 \%)$ who had synchronous unresectable liver metastases. These six patients showed good prognosis, with an MST of 22 months. DCS treatment led to complete remission of the peritoneal metastasis in nine patients $(9 / 33,27.3 \%)$, all of whom subsequently underwent surgery, achieving an MST of 28 months.

\section{Multivariate analysis of clinical factors affecting the postoperative outcome}

Univariate analyses revealed that downstaging $(P=0.012)$, R0 resection $(P=0.006)$, and pathological response $(P=0.006)$ affected survival among patients who underwent conversion therapy $(n=33)$. Other variables, such as the number of preoperative DCS courses and postoperative chemotherapy, were not significant prognostic factors. Multivariate analysis showed that pathological response $(P=0.009)$ was the only independent prognostic factor for conversion therapy (Table 4).

\section{Discussion}

Recently, conversion therapy for gastric cancer has attracted considerable attention as a new therapeutic strategy [12]. However, evidence regarding whether conversion therapy can produce considerable survival benefits for patients with metastatic gastric cancer is lacking. To date, few reports of conversion therapy in gastric cancer 

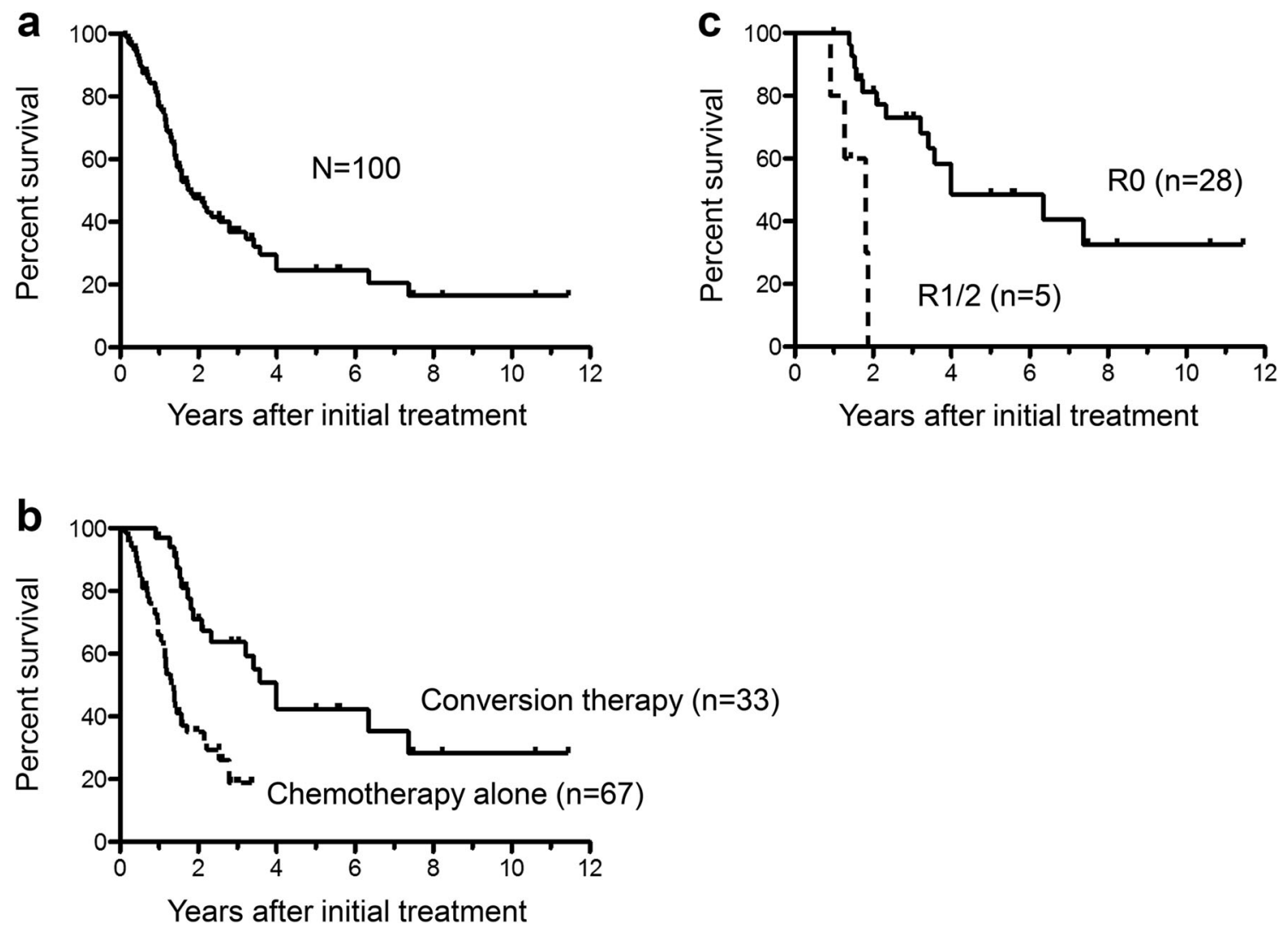

Fig. 2 Overall survival curves of patients with unresectable gastric cancer after initial chemotherapy. a Overall survival curves of 100 patients. b Differences in survival between those who underwent

conversion therapy (thick curve) and those who underwent chemotherapy alone (dotted curve). c Differences in survival between the $\mathrm{R} 0$ (thick curve) and $\mathrm{R} 1 / \mathrm{R} 2$ resection groups (dotted curve)
Table 4 Factors affecting survival among patients with conversion therapy

\begin{tabular}{|c|c|c|c|c|c|c|c|}
\hline \multirow[t]{2}{*}{ Factors } & \multirow[t]{2}{*}{$n$} & \multicolumn{3}{|c|}{ Univariate analysis } & \multicolumn{3}{|c|}{ Multivariate analysis } \\
\hline & & Hazard ratio & $95 \% \mathrm{CI}$ & $P$ value & Hazard ratio & $95 \% \mathrm{CI}$ & $P$ value \\
\hline \multicolumn{8}{|c|}{ Preoperative DCS (course) } \\
\hline $4 \geqq$ & 24 & 1 & & & 1 & & \\
\hline $5 \leqq$ & 9 & 2.911 & $0.985-16.87$ & 0.058 & 1.378 & $0.514-3.697$ & 0.524 \\
\hline \multicolumn{8}{|c|}{ Downstaging } \\
\hline No & 10 & 1 & & & 1 & & \\
\hline Yes & 23 & 0.264 & $0.028-0.613$ & 0.012 & 0.622 & $0.16-2.483$ & 0.521 \\
\hline \multicolumn{8}{|c|}{$\mathrm{R} 0$ resection } \\
\hline No & 5 & 1 & & & 1 & & \\
\hline Yes & 28 & 0.213 & $0.009-0.430$ & 0.006 & 0.398 & $0.124-1.275$ & 0.121 \\
\hline \multicolumn{8}{|c|}{ Pathological response } \\
\hline $0,1 \mathrm{a}$ & 7 & 1 & & & 1 & & \\
\hline $1 b, 2,3$ & 26 & 0.221 & $0.039-0.551$ & 0.006 & 0.139 & $0.031-0.617$ & 0.009 \\
\hline \multicolumn{8}{|c|}{ Postoperative chemotherapy } \\
\hline No & 5 & 1 & & & 1 & & \\
\hline Yes & 28 & 2.610 & $0.476-8.807$ & 0.339 & 3.170 & $0.386-25.974$ & 0.283 \\
\hline
\end{tabular}

CI confidence interval 
exist, because this cancer is usually accompanied by diverse metastatic lesions, including liver, peritoneal, and LN lesions, and because no effective chemotherapy enables significant responses for metastatic lesions; thus, complete resection has been identified.

Some retrospective analyses using S-1-based doublet regimens have reported good prognoses in conversion cases. Ishigami et al. reported the results of secondary gastrectomy in 18 patients using a combination of paclitaxel and S-1 [22]. The MST of the total 18 patients was 772 days, while the MST of the 13 patients who received R0 gastrectomy was 997 days. Kanda et al. described 28 stage IV gastric cancer patients who underwent surgery with curative intent after S-1-based chemotherapy, mainly S-1 plus cisplatin, and found that the 1-, 3-, and 5-year OS rates were 96.4, 53.3, and $34.3 \%$, respectively, with an MST of 37 months [14]. Moreover, Fukuchi et al. discussed 151 unresectable gastric cancer patients who received combination chemotherapy with $\mathrm{S}-1$ plus cisplatin or paclitaxel, of whom 40 (26\%) underwent conversion surgery; the MST for these 40 patients was 53 months [13].

We have previously reported that the DCS regimen showed a very high response rate $(87.1 \%)$ in patients with unresectable advanced gastric cancer [16, 17]. Another phase II study of DCS with a different treatment regimen also showed that this combination was highly effective (response rate, $81 \%$ ) [23]. Moreover, our phase II study of neoadjuvant DCS chemotherapy (2-4 cycles of DCS before surgery) for locally advanced resectable gastric cancer showed that the proportion of R0 resections in 43 eligible patients was $90.7 \%$, and pathological response was obtained in $65.9 \%$ [18], showing a much better therapeutic effect than other S-1-based regimens [24]. Therefore, DCS might be an appropriate regimen for conversion therapy.

In this study, we found that $33 / 100$ patients (33\%) were able to undergo conversion therapy. The proportion of R0 resections in the 33 resected patients was $84.8 \%$, which was supported by the fact that our regimen had a high pathological response rate of $78.8 \%$. This high rate may have contributed to a relatively good prognosis, with an MST of 47.8 months and 5-year OS of $42.4 \%$ in the conversion therapy cases. Moreover, we also demonstrated that conversion therapy could offer the possibility of 5-year long-term survival in $30.3 \%(10 / 33)$ of patients, while there were no survivors at 5 years in non-conversion cases. Thus, overall $10 \%(10 / 100)$ of patients with primarily unresectable metastases might be cured after DCS chemotherapy. This observation is important because these patients were originally considered for palliative chemotherapy, without any hope of long-term survival. Particularly, we found that conversion therapy was performed more often in patients with a good performance status or only one incurable factor (Table 2), indicating that such patients may represent potentially beneficial candidates for conversion therapy among all patients treated with the DCS regimen.

The degree of toxicity of preoperative chemotherapy is a critical problem because of potential adverse effects on operative morbidity and mortality. The DCS regimen was associated with a high incidence $(72.7 \%)$ of severe neutropenia in conversion therapy cases. However, febrile neutropenia occurred in only $6.1 \%$ of cases, which were transient, manageable with granulocyte-colony stimulating factor administration, and underwent dose reductions, preventing recurrence of toxicity. Obviously, DCS treatment necessitates careful observation of toxicity patterns to prevent treatment-associated toxicities. As to postoperative complications, the incidence of complications (24.2\%) was similar to that in patients undergoing conventional radical surgery for gastric cancer, such as a complication incidence of $20.9 \%$ in patients with D2 LN dissection and $28.1 \%$ in patients undergoing extended operation with aortic LN dissection (JCOG9501) [25]. Taken together, these findings suggest that conversion therapy after DCS therapy is safe and feasible.

Patients with unresectable gastric cancers can be classified into the following groups: (1) solid organ (e.g., liver and lungs) metastases group, (2) peritoneal metastases group, (3) LN metastases group, and (4) T4 invasion to an adjacent organ group.

In general, group 3 or 4 patients with only one group of metastasis sites are potentially good candidates for resection, with major response to chemotherapy. In this study, DCS was found to be especially effective for LN metastases (group 3), which showed a good conversion rate with the MST not yet reached. Despite no consensus on whether distant LN involvement such as para-aortic lymph node (PAN) metastasis limited to no. 16a2/b1 (JGCA classification) should be regarded as resectable disease, some researchers have treated PAN metastasis as resectable disease and a neoadjuvant strategy target [26]. In fact, among the nine conversion cases with distant LN metastasis (the only incurable factor), seven patients had no. 16a2/b1 LN involvement. Therefore, the efficacy of conversion therapy might have been overestimated, including in cases with relatively limited PAN metastases. In unresectable T4 lesions including pancreas, colon, and liver invasion (group 4), we found a relatively high conversion rate of $50 \%$ (7/ 14) and good survival (MST $76 \mathrm{M}$ ).

Conversely, group 1 and 2 patients falling in more than one metastasis group are generally considered truly inoperable or for a palliative setting. For group 1 patients, the prognosis of gastric cancer with concomitant liver metastasis is poor with a 5-year survival of $<10 \%$ [27, 28]. Despite curative hepatectomy, recurrence was previously reported in $62 \%$ of patients, with most cases developing 
intrahepatic recurrence [29], suggesting the presence of occult intrahepatic metastases even at the time of hepatectomy. Taken together, these results indicate the difficulty of obtaining a surgical cure in patients with hepatic metastases and the necessity of effective chemotherapy to eliminate possible metastases [30]. In the current study, conversion cases with liver metastases showed good prognosis, with an MST of 22 months, suggesting that patients can obtain survival benefits from this treatment. In group 2 patients, peritoneal metastasis is the most common non-curative factor for gastric cancer and is known to be relatively resistant to systemic chemotherapy. In fact, the MST in patients with peritoneal metastasis was reportedly only about 10 months in the JCOG 0106 study, using 5-FU plus methotrexate or 5-FU alone [31]. The DCS regimen, in which three effective drugs are administered as frontline treatment, is considered to be effective and feasible for the treatment of patients with peritoneal metastasis, because $\mathrm{S}-1$ contains a dihydropyrimidine dehydrogenase inhibitor, which may be preferable for the treatment of peritoneal metastases, as these are commonly associated with high dihydropyrimidine dehydrogenase activity [32]. In addition, docetaxel has favorable characteristics such as high efficacy against diffuse-type adenocarcinoma and a high rate of transition into the peritoneal cavity for the treatment of peritoneal metastasis [33]. In fact, DCS treatment resulted in survival comparable to intravenous and intraperitoneal paclitaxel administration of oral S-1 therapy, which showed an MST of 26.4 months for conversion cases in a previous study [34].

In Japan, postoperative adjuvant chemotherapy with S-1 is widely accepted as a standard treatment for patients with operable locally advanced gastric cancer. However, there is no consensus about the appropriate chemotherapeutic regimen, schedule, or duration of treatment for adjuvant chemotherapy after conversion therapy. In this study, 28/33 patients $(84.8 \%)$ received adjuvant chemotherapy, while $5 / 33$ patients who achieved pathological complete response did not undergo adjuvant chemotherapy. Most patients received S-1 alone, while ten patients received a doublet regimen such as docetaxel plus S-1 or cisplatin plus S-1 as adjuvant chemotherapy, considering the patients' tolerability and pathological response. In the ACTS-GC trial, $66.4 \%$ of patients received S- 1 alone for 1 year [35], while in the present study, we found that $66.7 \%$ of patients continued to receive S-1 for 1 year, suggesting the feasibility of adjuvant S-1 therapy in conversion cases. Although adjuvant chemotherapy was not a significant prognostic factor in this study, further prospective studies may be needed to demonstrate whether adjuvant chemotherapy is required after conversion therapy.

An important objective of our study was to define which patients would benefit the most from conversion therapy.
Factors affecting survival among patients with conversion therapy in the univariate analyses included downstaging, $\mathrm{R} 0$ resection, and pathological response, with only the pathological response showing statistical significance in multivariate analysis, as reported in other retrospective studies [24] (Table 4).

This study is limited by its retrospective and exploratory nature. Moreover, our cohort had a relatively small population of patients with multiple metastatic sites. As conversion therapy was performed more often in patients with only one incurable factor, the possibility of conversion therapy could be overestimated in this cohort. The role of surgical resection in patients with complete or near complete response is debatable. Even in conversion cases with good response to DCS chemotherapy, residual cancer had to be histologically confirmed in most cases. Therefore, surgery may have contributed to improved overall survival. However, our results did not necessarily demonstrate that surgical resection per se improved final outcomes. Nevertheless, we demonstrate that surgery following preoperative DCS chemotherapy could be associated with a high complete resection rate and long-term survival, particularly in patients with a good pathological response.

Taken together, these results justify the need for a largescale randomized prospective study to determine whether conversion therapy leads to a better prognosis and to investigate the therapeutic usefulness of conversion therapy using DCS chemotherapy.

\section{Compliance with ethical standards}

Human rights statement and informed consent All procedures followed were in accordance with the ethical standards of the responsible committee on human experimentation (institutional and national) and with the Helsinki Declaration of 1964 and later versions. Informed consent or substitute for it was obtained from all patients for being included in the study.

Conflict of interest The authors declare that they have no conflict of interest.

\section{References}

1. Torre LA, Bray F, Siegel RL, Ferlay J, Lortet-Tieulent J, Jemal A. Global cancer statistics, 2012. CA Cancer J Clin. 2015;65:87-108.

2. Saka M, Morita S, Fukagawa T, Katai H. Present and future status of gastric cancer surgery. Jpn J Clin Oncol. 2011;41:307-13.

3. Alberts SR, Cervantes A, van de Velde CJH. Gastric cancer: epidemiology, pathology and treatment. Ann Oncol. 2003;14 Suppl 2:ii31-6.

4. Shah MA. Update on metastatic gastric and esophageal cancers. J Clin Oncol. 2015;33:1760-9.

5. Yoshida M. Long-term survival and prognostic factors in patients with metastatic gastric cancers treated with chemotherapy in the Japan clinical oncology group (JCOG) study. Jpn J Clin Oncol. 2004;34:654-9. 
6. Ohtsu A. Chemotherapy for metastatic gastric cancer: past, present, and future. J Gastroenterol. 2008;43:256-64.

7. Van Cutsem E, Moiseyenko VM, Tjulandin S, Majlis A, Constenla M, Boni C, et al. Phase III study of docetaxel and cisplatin plus fluorouracil compared with cisplatin and fluorouracil as firstline therapy for advanced gastric cancer: a report of the V325 study group. J Clin Oncol. 2006;24:4991-7.

8. Cunningham D, Starling N, Rao S, Iveson T, Nicolson M, Coxon $\mathrm{F}$, et al. Capecitabine and oxaliplatin for advanced esophagogastric cancer. N Engl J Med. 2008;358:36-46.

9. Koizumi W, Narahara H, Hara T, Takagane A, Akiya T, Takagi $\mathrm{M}$, et al. S-1 plus cisplatin versus S-1 alone for first-line treatment of advanced gastric cancer (SPIRITS trial): a phase III trial. Lancet Oncol. 2008;9:215-21.

10. Adam R, Delvart V, Pascal G, Valeanu A, Castaing D, Azoulay $\mathrm{D}$, et al. Rescue surgery for unresectable colorectal liver metastases downstaged by chemotherapy: a model to predict long-term survival. Ann Surg. 2004;240:644-57.

11. Adam R, Wicherts DA, de Haas RJ, Ciacio O, Levi F, Paule B, et al. Patients with initially unresectable colorectal liver metastases: is there a possibility of cure? J Clin Oncol. 2009;27:1829-35.

12. Yoshida K, Yamaguchi K, Okumura N, Tanahashi T, Kodera Y. Is conversion therapy possible in stage IV gastric cancer: the proposal of new biological categories of classification. Gastric Cancer. 2016;19:329-38.

13. Fukuchi M, Ishiguro T, Ogata K, Suzuki O, Kumagai Y, Ishibashi $\mathrm{K}$, et al. Prognostic role of conversion surgery for unresectable gastric cancer. Ann Surg Oncol. 2015;22:3618-24.

14. Kanda T, Yajima K, Kosugi S-I, Ishikawa T, Ajioka Y, Hatakeyama K. Gastrectomy as a secondary surgery for stage IV gastric cancer patients who underwent S-1-based chemotherapy: a multiinstitute retrospective study. Gastric Cancer. 2011;15:235-44.

15. Yoshida K, Yamaguchi K, Okumura N, Osada S, Takahashi T, Tanaka Y, et al. The roles of surgical oncologists in the new era: minimally invasive surgery for early gastric cancer and adjuvant surgery for metastatic gastric cancer. Pathobiology. 2011;78: $343-52$.

16. Takayama T, Sato Y, Sagawa T, Okamoto T, Nagashima H, Takahashi Y, et al. Phase I study of S-1, docetaxel and cisplatin combination chemotherapy in patients with unresectable metastatic gastric cancer. Br J Cancer. 2007;97:851-6.

17. Sato Y, Takayama T, Sagawa T, Takahashi Y, Ohnuma H, Okubo $\mathrm{S}$, et al. Phase II study of S-1, docetaxel and cisplatin combination chemotherapy in patients with unresectable metastatic gastric cancer. Cancer Chemother Pharmacol. 2009;66:721-8.

18. Hirakawa M, Sato Y, Ohnuma H, Takayama T, Sagawa T, Nobuoka T, et al. A phase II study of neoadjuvant combination chemotherapy with docetaxel, cisplatin, and S-1 for locally advanced resectable gastric cancer: nucleotide excision repair (NER) as potential chemoresistance marker. Cancer Chemother Pharmacol. 2013;71:789-97.

19. Japanese Gastric Cancer Association. Japanese classification of gastric carcinoma-2nd english edition. Gastric Cancer. 1998;1: $10-24$.

20. Japanese Gastric Cancer Association. Japanese classification of gastric carcinoma-2nd english edition-response assessment of chemotherapy and radiotherapy for gastric carcinoma: clinical criteria. Gastric Cancer. 2001;4:1-8.

21. Japanese Gastric Cancer Association. Japanese classification of gastric carcinoma: 3rd english edition. Gastric Cancer. 2011;14: 101-12.
22. Ishigami S, Natsugoe S, Nakajo A, Matsumoto M, Uenosono Y, Arigami T, et al. Salvage Gastrectomy Following a Combination of Biweekly Paclitaxel and S-1 for Stage IV Gastric Cancer. J Gastrointest Surg. 2008;12:1370-5.

23. Koizumi W, Nakayama N, Tanabe S, Sasaki T, Higuchi K, Nishimura K, et al. A multicenter phase II study of combined chemotherapy with docetaxel, cisplatin, and S-1 in patients with unresectable or recurrent gastric cancer (KDOG 0601). Cancer Chemother Pharmacol. 2011;69:407-13.

24. Kurokawa Y, Shibata T, Sasako M, Sano T, Tsuburaya A, Iwasaki $\mathrm{Y}$, et al. Validity of response assessment criteria in neoadjuvant chemotherapy for gastric cancer (JCOG0507-A). Gastric Cancer. 2014;17:514-21.

25. Sano T, Sasako M, Yamamoto S, Nashimoto A, Kurita A, Hiratsuka M, et al. Gastric cancer surgery: morbidity and mortality results from a prospective randomized controlled trial comparing D2 and extended para-aortic lymphadenectomy-Japan clinical oncology group study 9501. J Clin Oncol. 2004;22: 2767-73.

26. Tsuburaya A, Mizusawa J, Tanaka T, Fukushima N, Nashimoto A, Sasako M, et al. Neoadjuvant chemotherapy with S-1 and cisplatin followed by D2 gastrectomy with para-aortic lymph node dissection for gastric cancer with extensive lymph node metastasis. Br J Surg. 2014;101:653-60.

27. Kakeji Y, Morita M, Maehara Y. Strategies for treating liver metastasis from gastric cancer. Surg Today. 2010;40:287-94.

28. Okano K, Maeba T, Ishimura K, Karasawa Y, Goda F, Wakabayashi $\mathrm{H}$, et al. Hepatic resection for metastatic tumors from gastric cancer. Ann Surg. 2002;235:86-91.

29. Sakamoto Y, Sano T, Shimada K, Esaki M, Saka M, Fukagawa T, et al. Favorable indications for hepatectomy in patients with liver metastasis from gastric cancer. J Surg Oncol. 2007;95:534-9.

30. Grimes N, Devlin J, Dunne DFJ, Poston G, Fenwick S, Malik H. The role of hepatectomy in the management of metastatic gastric adenocarcinoma: a systematic review. Surg Oncol. 2014;23: 177-85.

31. Shirao K, Boku N, Yamada Y, Yamaguchi K, Doi T, Goto M, et al. Randomized Phase III study of 5-fluorouracil continuous infusion vs. sequential methotrexate and 5-fluorouracil therapy in far advanced gastric cancer with peritoneal metastasis (JCOG0106). Jpn J Clin Oncol. 2013;43:972-80.

32. Usuki H, Ishimura $K$, Yachida $S$, Hagiike M, Okano K, Izuishi K, et al. Dihydropyrimidine dehydrogenase (DPD) activity in gastric cancer tissue and effect of DPD inhibitory fluoropyrimidines. Gastric Cancer. 2003;6(Suppl 1):66-70.

33. Tamegai H, Kaiga T, Kochi M, Fujii M, Kanamori N, Mihara Y, et al. Pharmacokinetics of docetaxel in gastric cancer patients with malignant ascites. Cancer Chemother Pharmacol. 2013;71:727-31.

34. Kitayama J, Ishigami $\mathrm{H}$, Yamaguchi $\mathrm{H}$, Yamashita $\mathrm{H}$, Emoto $\mathrm{S}$, Kaisaki $S$, et al. Salvage gastrectomy after intravenous and intraperitoneal paclitaxel (PTX) administration with oral S-1 for peritoneal dissemination of advanced gastric cancer with malignant ascites. Ann Surg Oncol. 2014;21:539-46.

35. Sakuramoto S, Sasako M, Yamaguchi T, Kinoshita T, Fujii M, Nashimoto A, et al. Adjuvant chemotherapy for gastric cancer with S-1, an oral fluoropyrimidine. $\mathrm{N}$ Engl $\mathrm{J}$ Med. 2007:357:1810-20. 